\title{
Strong convergence with a modified iterative projection method for hierarchical fixed point problems and variational inequalities
}

\author{
Ibrahim Karahan ${ }^{1}$ and Murat Ozdemir ${ }^{2}$ \\ ${ }^{1}$ Department of Mathematics, Erzurum Technical University, Erzurum, Turkey \\ ${ }^{2}$ Department of Mathematics, Ataturk University, Erzurum, Turkey.
}

Received: 8 September 2015, Revised: 11 September 2015, Accepted: 16 September 2015

Published online: 21 April 2016.

\begin{abstract}
Let $C$ be a nonempty closed convex subset of a real Hilbert space $H$. Let $\left\{T_{n}\right\}: C \rightarrow H$ be a sequence of nearly nonexpansive mappings such that $\mathfrak{F}:=\bigcap_{i=1}^{\infty} F\left(T_{i}\right) \neq \varnothing$. Let $V: C \rightarrow H$ be a $\gamma$-Lipschitzian mapping and $F: C \rightarrow H$ be a $L$-Lipschitzian and $\eta$-strongly monotone operator. This paper deals with a modified iterative projection method for approximating a solution of the hierarchical fixed point problem. It is shown that under certain approximate assumptions on the operators and parameters, the modified iterative sequence $\left\{x_{n}\right\}$ converges strongly to $x^{*} \in \mathfrak{F}$ which is also the unique solution of the following variational inequality:

$$
\left\langle(\rho V-\mu F) x^{*}, x-x^{*}\right\rangle \leq 0, \forall x \in \mathfrak{F} .
$$

As a special case, this projection method can be used to find the minimum norm solution of above variational inequality; namely, the unique solution $x^{*}$ to the quadratic minimization problem: $x^{*}=\operatorname{argmin}_{x \in \mathfrak{F}}\|x\|^{2}$. The results here improve and extend some recent corresponding results of other authors.
\end{abstract}

Keywords: Variational inequality, hierarchical fixed point, nearly nonexpansive mappings, strong convergence.

\section{Introduction}

Throughout this paper, we assume that $H$ is a real Hilbert space whose inner product and norm are denoted by $\langle\cdot, \cdot\rangle$ and $\|\cdot\|$, respectively, and $C$ is a nonempty closed convex subset of $H$. The set of fixed points of a mapping $T$ is denoted by Fix $(T)$, that is, Fix $(T)=\{x \in H: T x=x\}$. Below we gather some basic definitions and results which are needed in the subsequent sections. Recall that a mapping $T: C \rightarrow H$ is called $L$-Lipschitzian if there exits a constant $L>0$ such that $\|T x-T y\| \leq L\|x-y\|, \forall x, y \in C$. In particular, if $L \in[0,1)$, then $T$ is said to be a contraction; if $L=1$, then $T$ is called a nonexpansive mapping. $T$ is called nearly nonexpansive [1,2] with respect to a fixed sequence $\left\{a_{n}\right\}$ in $[0, \infty)$ with $a_{n} \rightarrow 0$ if $\left\|T^{n} x-T^{n} y\right\| \leq\|x-y\|+a_{n}, \forall x, y \in C$ and $n \geq 1$.

A mapping $F: C \rightarrow H$ is called $\eta$-strongly monotone if there exists a constant $\eta \geq 0$ such that

$$
\langle F x-F y, x-y\rangle \geq \eta\|x-y\|^{2}, \forall x, y \in C .
$$

In particular, if $\eta=0$, then $F$ is said to be monotone.

It is well known that for any $x \in H$, there exists a unique point $y_{0} \in C$ such that

$$
\left\|x-y_{0}\right\|=\inf \{\|x-y\|: y \in C\}
$$


where $C$ is a nonempty closed convex subset of $H$. We denote $y_{0}$ by $P_{C} x$, where $P_{C}$ is called the metric projection of $H$ onto $C$. It is easy to see $P_{C}$ is a nonexpansive mapping.

Let $S: C \rightarrow H$ be a nonexpansive mapping. The following problem is called a hierarchical fixed point problem: Find $x^{*} \in \operatorname{Fix}(T)$ such that

$$
\left\langle x^{*}-S x^{*}, x-x^{*}\right\rangle \geq 0, x \in F i x(T) .
$$

The problem (1) is equivalent to the following fixed point problem: to find an $x^{*} \in C$ that satisfies $x^{*}=P_{F i x(T)} S x^{*}$. We know that $F i x(T)$ is closed and convex, so the metric projection $P_{F i x(T)}$ is well defined.

It is known that the hierarchical fixed point problem (1) links with some monotone variational inequalities and convex programming problems; see $[3,4,5,6,7,8]$. Various methods have been proposed to solve the hierarchical fixed point problem; see Moudafi in [10], Mainge and Moudafi in [11], Yao and Liou in [12], Xu in [13], Marino and Xu in [14] and Bnouhachem and Noor in [15].

In 2006, Marino and Xu [16] introduced the viscosity iterative method for nonexpansive mappings. They considered the following general iterative method:

$$
x_{n+1}=\alpha_{n} \gamma f\left(x_{n}\right)+\left(1-\alpha_{n} A\right) T x_{n}, \forall n \geq 0,
$$

where $f$ is a contraction, $T$ is a nonexpansive mapping and $A$ is a strongly positive bounded linear operator on $H$; that is, there is a constant $\gamma>0$ such that $\langle A x, x\rangle \geq \gamma\|x\|, \forall x \in H$. They proved that the sequence $\left\{x_{n}\right\}$ generated by (2) converges strongly to the unique solution of the variational inequality

$$
\left\langle(\gamma f-A) x^{*}, x-x^{*}\right\rangle \leq 0, \forall x \in C
$$

which is the optimality condition for the minimization problem

$$
\min _{x \in C} \frac{1}{2}\langle A x, x\rangle-h(x)
$$

where $h$ is a potential function for $\gamma f$, i.e., $h^{\prime}(x)=\gamma f(x)$ for all $x \in H$.

On the other hand, in 2010, Tian [4] proposed an implicit and an explicit schemes on combining the iterative methods of Yamada [9] and Marino and $\mathrm{Xu}$ [16]. He also proved the strong convergence of these two schemes to a fixed point of a nonexpansive mapping $T$ defined on a real Hilbert space under suitable conditions. In the same year, Ceng et al. [17] investigated the following iterative method:

$$
x_{n+1}=P_{C}\left[\alpha_{n} \rho V x_{n}+\left(1-\alpha_{n} \mu F\right) T x_{n}\right], \forall n \geq 0,
$$

where $F$ is a $L$-Lipschitzian and $\eta$-strongly monotone operator with constants $L, \eta>0$ and $V$ is a $\gamma$-Lipschitzian (possibly non-self) mapping with constant $\gamma \geq 0$ such that $0<\mu<\frac{2 \eta}{L^{2}}$ and $0 \leq \rho \gamma<1-\sqrt{1-\mu\left(2 \eta-\mu L^{2}\right)}$. They proved that under some approximate assumptions on the operators and parameters, the sequence $\left\{x_{n}\right\}$ generated by (4) converges strongly to the unique solution of the variational inequality

$$
\left\langle(\rho V-\mu F) x^{*}, x-x^{*}\right\rangle \leq 0, \forall x \in F i x(T) .
$$

Fix a sequence $\left\{a_{n}\right\}$ in $[0, \infty)$ with $a_{n} \rightarrow 0$ and let $\left\{T_{n}\right\}$ be a sequence of mappings from $C$ into $H$. Then, the sequence $\left\{T_{n}\right\}$ is called a sequence of nearly nonexpansive mappings $[18,19]$ with respect to a sequence $\left\{a_{n}\right\}$ if

$$
\left\|T_{n} x-T_{n} y\right\| \leq\|x-y\|+a_{n}, \forall x, y \in C, \forall n \geq 1 .
$$


It is obvious that the sequence of nearly nonexpansive mappings is a wider class of sequence of nonexpansive mappings. Recently, in 2012, Sahu et al. [19] introduced the following iterative process for the sequence of nearly nonexpansive mappings $\left\{T_{n}\right\}$ defined by (6)

$$
x_{n+1}=P_{C}\left[\alpha_{n} \rho V x_{n}+\left(1-\alpha_{n} \mu F\right) T_{n} x_{n}\right], \forall n \geq 1 .
$$

They proved that the sequence $\left\{x_{n}\right\}$ generated by (7) converges strongly to the unique solution of the variational inequality (5).

Very recently, in 2013, Wang and Xu [20] investigated an iterative method for a hierarchical fixed point problem by

$$
\left\{\begin{array}{l}
y_{n}=\beta_{n} S x_{n}+\left(1-\beta_{n}\right) x_{n} \\
x_{n+1}=P_{C}\left[\alpha_{n} \rho V x_{n}+\left(I-\alpha_{n} \mu F\right) T y_{n}\right], \forall n \geq 0
\end{array}\right.
$$

where $S: C \rightarrow C$ is a nonexpansive mapping. They proved that under some approximate assumptions on the operators and parameters, the sequence $\left\{x_{n}\right\}$ generated by (8) converges strongly to the unique solution of the variational inequality (5). In addition to all these methods, similar methods are considered in several papers, see [24, $25,26,27,28]$.

In this paper, motivated by the work of Wang and $\mathrm{Xu}$ [20] and Sahu et al. [19] and by the recent work going in this direction, we introduce a modified iterative projection method and prove a strong convergence theorem based on this method for computing an element of the set of common fixed points of a sequence $\left\{T_{n}\right\}$ of nearly nonexpansive mappings defined by (6) which is also an unique solution of the variational inequality (5). The presented method improves and generalizes many known results for solving variational inequality problems and hierarchical fixed point problems, see, e.g., $[4,16,17,19,20]$ and relevant references cited therein.

\section{Preliminaries}

Let $\left\{x_{n}\right\}$ be a sequence in a Hilbert space $H$ and $x \in H$. Throughout this paper, $x_{n} \rightarrow x$ denotes that $\left\{x_{n}\right\}$ strongly converges to $x$ and $x_{n} \rightarrow x$ denotes that $\left\{x_{n}\right\}$ weakly converges to $x$.

Let $C$ be a nonempty subset of a real Hilbert space $H$ and $T_{1}, T_{2}: C \rightarrow H$ be two mappings. We denote $\mathscr{B}(C)$, the collection of all bounded subsets of $C$. The deviation between $T_{1}$ and $T_{2}$ on $B \in \mathscr{B}(C)$, denoted by $\mathfrak{D}_{B}\left(T_{1}, T_{2}\right)$, is defined by

$$
\mathfrak{D}_{B}\left(T_{1}, T_{2}\right)=\sup \left\{\left\|T_{1} x-T_{2} x\right\|: x \in B\right\} .
$$

The following lemmas will be used in the next section.

Lemma 1. [18] Let $C$ be a nonempty closed bounded subset of a Banach space $X$ and $\left\{T_{n}\right\}$ be a sequence of nearly nonexpansive self-mappings on $C$ with a sequence $\left\{a_{n}\right\}$ such that $\mathfrak{D}_{C}\left(T_{n}, T_{n+1}\right)<\infty$. Then, for each $x \in C,\left\{T_{n} x\right\}$ converges strongly to some point of C. Moreover, if T is a mapping from $C$ into itself defined by $T z=\lim _{n \rightarrow \infty} T_{n} z$ for all $z \in C$, then $T$ is nonexpansive and $\lim _{n \rightarrow \infty} \mathfrak{D}_{C}\left(T_{n}, T\right)=0$.

Lemma 2. [17] Let $V: C \rightarrow H$ be a $\gamma$-Lipschitzian mapping with a constant $\gamma \geq 0$ and let $F: C \rightarrow H$ be a L-Lipschitzian and $\eta$-strongly monotone operator with constants $L, \eta>0$. Then for $0 \leq \rho \gamma<\mu \eta$,

$$
\langle(\mu F-\rho V) x-(\mu F-\rho V) y, x-y\rangle \geq(\mu \eta-\rho \gamma)\|x-y\|^{2}, \forall x, y \in C
$$

That is, $\mu F-\rho V$ is strongly monotone with coefficient $\mu \eta-\rho \gamma$. 
Lemma 3. [9] Let $C$ be a nonempty subset of a real Hilbert space $H$. Suppose that $\lambda \in(0,1)$ and $\mu>0$. Let $F: C \rightarrow H$ be a $L$-Lipschitzian and $\eta$-strongly monotone operator on $C$. Define the mapping $G: C \rightarrow H$ by

$$
G x=x-\lambda \mu F x, \forall x \in C \text {. }
$$

Then, $G$ is a contraction that provided $\mu<\frac{2 \eta}{L^{2}}$. More precisely, for $\mu \in\left(0, \frac{2 \eta}{L^{2}}\right)$,

$$
\|G x-G y\| \leq(1-\lambda v)\|x-y\|, \forall x, y \in C,
$$

where $v=1-\sqrt{1-\mu\left(2 \eta-\mu L^{2}\right)}$.

Lemma 4. [21] Let $C$ be a nonempty closed convex subset of a real Hilbert space $H$, and $T$ be a nonexpansive selfmapping on C. If Fix $(T) \neq \varnothing$, then $I-T$ is demiclosed; that is whenever $\left\{x_{n}\right\}$ is a sequence in $C$ weakly converging to some $x \in C$ and the sequence $\left\{(I-T) x_{n}\right\}$ strongly converges to some $y$, it follows that $(I-T) x=y$. Here I is the identity operator of $H$.

Lemma 5. [22] Assume that $\left\{x_{n}\right\}$ is a sequence of nonnegative real numbers satisfying the conditions

$$
x_{n+1} \leq\left(1-\alpha_{n}\right) x_{n}+\alpha_{n} \beta_{n}, \forall n \geq 1
$$

where $\left\{\alpha_{n}\right\}$ and $\left\{\beta_{n}\right\}$ are sequences of real numbers such that

(i) $\left\{\alpha_{n}\right\} \subset[0,1]$ and $\sum_{n=1}^{\infty} \alpha_{n}=\infty$

(ii) $\limsup _{n \rightarrow \infty} \beta_{n} \leq 0$.

Then $\lim _{n \rightarrow \infty} x_{n}=0$.

\section{Main results}

Now, we give the main results in this paper.

Theorem 1. Let $C$ be a nonempty closed convex subset of a real Hilbert space $H$. Let $S: C \rightarrow H$ be a nonexpansive mapping and $\left\{T_{n}\right\}$ be a sequence of nearly nonexpansive mappings with the sequence $\left\{a_{n}\right\}$ such that $F:=\cap_{n=1}^{\infty} F i x\left(T_{n}\right) \neq \varnothing$. Suppose that $T x=\lim _{n \rightarrow \infty} T_{n} x$ for all $x \in C$ and $F i x(T)=F$. Let $V: C \rightarrow H$ be a $\gamma$-Lipschitzian mapping, $F: C \rightarrow H$ be a L-Lipschitzian and $\eta$-strongly monotone operator such that these coefficients satisfy $0<\mu<\frac{2 \eta}{L^{2}}, 0 \leq \rho \gamma<v$, where $v=1-\sqrt{1-\mu\left(2 \eta-\mu L^{2}\right)}$. For an arbitrarily initial value $x_{1}$, consider the sequence $\left\{x_{n}\right\}$ in $C$ generated by

$$
\left\{\begin{array}{l}
y_{n}=P_{C}\left[\beta_{n} S x_{n}+\left(1-\beta_{n}\right) x_{n}\right], \\
x_{n+1}=P_{C}\left[\alpha_{n} \rho V x_{n}+\left(I-\alpha_{n} \mu F\right) T_{n} y_{n}\right], n \geq 1,
\end{array}\right.
$$

where $\left\{\alpha_{n}\right\}$ and $\left\{\beta_{n}\right\}$ are sequences in $[0,1]$ satisfying the conditions:

(C1) $\lim _{n \rightarrow \infty} \alpha_{n}=0$ and $\sum_{n=1}^{\infty} \alpha_{n}=\infty$;

(C2) $\lim _{n \rightarrow \infty} \frac{a_{n}}{\alpha_{n}}=0, \lim _{n \rightarrow \infty} \frac{\beta_{n}}{\alpha_{n}}=0, \lim _{n \rightarrow \infty} \frac{\left|\alpha_{n}-\alpha_{n-1}\right|}{\alpha_{n}}=0$ and

$$
\lim _{n \rightarrow \infty} \frac{\left|\beta_{n}-\beta_{n-1}\right|}{\alpha_{n}}=0
$$

(C3) $\lim _{n \rightarrow \infty} \mathfrak{D}_{B}\left(T_{n}, T_{n+1}\right)=0$ and $\lim _{n \rightarrow \infty} \frac{\mathfrak{D}_{B}\left(T_{n}, T_{n+1}\right)}{\alpha_{n}}=0$ for each $B \in \mathscr{B}(C)$.

Then, the sequence $\left\{x_{n}\right\}$ converges strongly to $x^{*} \in \mathfrak{F}$, where $x^{*}$ is the unique solution of the variational inequality

$$
\left\langle(\rho V-\mu F) x^{*}, x-x^{*}\right\rangle \leq 0, \forall x \in \mathfrak{F}
$$


In particular, the point $x^{*}$ is the minimum norm fixed point of $T$, that is $x^{*}$ is the unique solution of the quadratic minimization problem

$$
x^{*}=\operatorname{argmin}_{x \in \mathfrak{F}}\|x\|^{2} .
$$

Proof. Since the mapping $T$ is defined by $T x=\lim _{n \rightarrow \infty} T_{n} x$ for all $x \in C$, by Lemma $1, T$ is a nonexpansive mapping, and $\operatorname{Fix}(T) \neq \varnothing$. Moreover, since the operator $\mu F-\rho V$ is $(\mu \eta-\rho \gamma)$-strongly monotone by Lemma 2 , we get the uniqueness of the solution of the variational inequality (10). Let denote this solution by $x^{*} \in F i x(T)=\mathfrak{F}$.

Now, we divide our proof into six steps.

Step 1. First we show that the sequences $\left\{x_{n}\right\}$ is bounded. From hypothesis (C2), without loss of generality, we may assume that $\beta_{n} \leq \alpha_{n}$, for $n \geq 1$. Hence, we get $\lim _{n \rightarrow \infty} \beta_{n}=0$. Let $p \in \mathfrak{F}$ and $t_{n}=\alpha_{n} \rho V x_{n}+\left(I-\alpha_{n} \mu F\right) T_{n} y_{n}$. Then we have

$$
\begin{aligned}
\left\|y_{n}-p\right\| & =\left\|P_{C}\left[\beta_{n} S x_{n}+\left(1-\beta_{n}\right) x_{n}\right]-P_{C} p\right\| \\
& \leq\left\|\beta_{n} S x_{n}+\left(1-\beta_{n}\right) x_{n}-p\right\| \\
& \leq\left(1-\beta_{n}\right)\left\|x_{n}-p\right\|+\beta_{n}\left\|S x_{n}-p\right\| \\
& \leq\left(1-\beta_{n}\right)\left\|x_{n}-p\right\|+\beta_{n}\left\|S x_{n}-S p\right\|+\beta_{n}\|S p-p\| \\
& \leq\left\|x_{n}-p\right\|+\beta_{n}\|S p-p\|,
\end{aligned}
$$

and

$$
\begin{aligned}
\left\|x_{n+1}-p\right\| & =\left\|P_{C} t_{n}-P_{C} p\right\| \\
& \leq\left\|t_{n}-p\right\| \\
& =\left\|\alpha_{n} \rho V x_{n}+\left(I-\alpha_{n} \mu F\right) T_{n} y_{n}-p\right\| \\
& \leq \alpha_{n}\left\|\rho V x_{n}-\mu F p\right\|+\left\|\left(I-\alpha_{n} \mu F\right) T_{n} y_{n}-\left(I-\alpha_{n} \mu F\right) T_{n} p\right\| \\
& \leq \alpha_{n} \rho \gamma\left\|x_{n}-p\right\|+\alpha_{n}\|\rho V p-\mu F p\| \\
& +\left(1-\alpha_{n} v\right)\left(\left\|y_{n}-p\right\|+a_{n}\right) .
\end{aligned}
$$

From (11) and (12), we get

$$
\begin{aligned}
\left\|x_{n+1}-p\right\| & \leq \alpha_{n} \rho \gamma\left\|x_{n}-p\right\|+\alpha_{n}\|\rho V p-\mu F p\| \\
& +\left(1-\alpha_{n} v\right)\left(\left\|x_{n}-p\right\|+\beta_{n}\|S p-p\|+a_{n}\right) \\
& \leq\left(1-\alpha_{n}(v-\rho \gamma)\right)\left\|x_{n}-p\right\|+\alpha_{n}\left(\|\rho V p-\mu F p\|+\|S p-p\|+a_{n}\right) \\
& \leq\left(1-\alpha_{n}(v-\rho \gamma)\right)\left\|x_{n}-p\right\| \\
& +\alpha_{n}(v-\rho \gamma)\left[\frac{1}{(v-\rho \gamma)}\left(\|\rho V p-\mu F p\|+\|S p-p\|+\frac{a_{n}}{\alpha_{n}}\right)\right] .
\end{aligned}
$$

Note that $\frac{a_{n}}{\alpha_{n}} \rightarrow 0$ as $n \rightarrow \infty$, so there exists a constant $M>0$ such that

$$
\|\rho V p-\mu F p\|+\|S p-p\|+\frac{a_{n}}{\alpha_{n}} \leq M, \forall n \geq 1 .
$$

Thus, from (13) we have

$$
\left\|x_{n+1}-p\right\| \leq\left(1-\alpha_{n}(v-\rho \gamma)\right)\left\|x_{n}-p\right\|+\alpha_{n}(v-\rho \gamma) \frac{M}{(v-\rho \gamma)}
$$


By induction, we get

$$
\left\|x_{n+1}-p\right\| \leq \max \left\{\left\|x_{1}-p\right\|, \frac{M}{(v-\rho \gamma)}\right\} .
$$

Hence, we obtain that $\left\{x_{n}\right\}$ is bounded. So, the sequences $\left\{y_{n}\right\},\left\{T x_{n}\right\},\left\{S x_{n}\right\},\left\{V x_{n}\right\}$ and $\left\{F T y_{n}\right\}$ are bounded.

Step 2. Now, we show that $\lim _{n \rightarrow \infty}\left\|x_{n+1}-x_{n}\right\|=0$. By using the iteration (9), we have

$$
\begin{aligned}
\left\|y_{n}-y_{n-1}\right\| & =\left\|P_{C}\left[\beta_{n} S x_{n}+\left(1-\beta_{n}\right) x_{n}\right]-P_{C}\left[\beta_{n-1} S x_{n-1}-\left(1-\beta_{n-1}\right) x_{n-1}\right]\right\| \\
& \leq \beta_{n}\left\|S x_{n}-S x_{n-1}\right\|+\left(1-\beta_{n}\right)\left\|x_{n}-x_{n-1}\right\| \\
& +\left|\beta_{n}-\beta_{n-1}\right|\left(\left\|S x_{n-1}\right\|+\left\|x_{n-1}\right\|\right) \\
& \leq\left\|x_{n}-x_{n-1}\right\|+\left|\beta_{n}-\beta_{n-1}\right| M_{1},
\end{aligned}
$$

where $M_{1}$ is a constant such that $\sup _{n \geq 1}\left\{\left\|S x_{n}\right\|+\left\|x_{n}\right\|\right\} \leq M_{1}$. Also, by using the inequality (14), we get

$$
\begin{aligned}
\left\|x_{n+1}-x_{n}\right\| & \leq\left\|P_{C} t_{n}-P_{C} t_{n-1}\right\| \\
& \leq \| \alpha_{n} \rho V x_{n}+\left(I-\alpha_{n} \mu F\right) T_{n} y_{n} \\
& -\alpha_{n-1} \rho V x_{n-1}+\left(I-\alpha_{n-1} \mu F\right) T_{n-1} y_{n-1} \| \\
& \leq \| \alpha_{n} \rho V\left(x_{n}-x_{n-1}\right)+\left(\alpha_{n}-\alpha_{n-1}\right) \rho V x_{n-1}+\left(I-\alpha_{n} \mu F\right) T_{n} y_{n} \\
& -\left(I-\alpha_{n} \mu F\right) T_{n} y_{n-1}+T_{n} y_{n-1}-T_{n-1} y_{n-1} \\
& +\alpha_{n-1} \mu F T_{n-1} y_{n-1}-\alpha_{n} \mu F T_{n} y_{n-1} \| \\
& \leq \alpha_{n} \rho \gamma\left\|x_{n}-x_{n-1}\right\|+\gamma\left|\alpha_{n}-\alpha_{n-1}\right|\left\|V x_{n-1}\right\| \\
& +\left(1-\alpha_{n} v\right)\left\|T_{n} y_{n}-T_{n} y_{n-1}\right\|+\left\|T_{n} y_{n-1}-T_{n-1} y_{n-1}\right\| \\
& +\mu\left\|\alpha_{n-1} F T_{n-1} y_{n-1}-\alpha_{n} F T_{n} y_{n-1}\right\| \\
& \leq \alpha_{n} \rho \gamma\left\|x_{n}-x_{n-1}\right\|+\gamma\left|\alpha_{n}-\alpha_{n-1}\right|\left\|V x_{n-1}\right\| \\
& +\left(1-\alpha_{n} v\right)\left[\left\|y_{n}-y_{n-1}\right\|+a_{n}\right]+\left\|T_{n} y_{n-1}-T_{n-1} y_{n-1}\right\| \\
& +\mu\left\|\alpha_{n-1}\left(F T_{n-1} y_{n-1}-F T_{n} y_{n-1}\right)-\left(\alpha_{n}-\alpha_{n-1}\right) F T_{n} y_{n-1}\right\| \\
& \leq \alpha_{n} \rho \gamma\left\|x_{n}-x_{n-1}\right\|+\gamma\left\|V x_{n-1}\right\|+\left(1-\alpha_{n} v\right)\left\|x_{n}-x_{n-1}\right\| \\
& +\left(1-\alpha_{n} v\right)\left|\beta_{n}-\beta_{n-1}\right| M_{1}+\left(1-\alpha_{n} \nu\right) a_{n}+\mathfrak{D}_{B}\left(T_{n}, T_{n-1}\right) \\
& +\mu \alpha_{n-1} L \mathfrak{D}_{B}\left(T_{n}, T_{n-1}\right)+\left|\alpha_{n}-\alpha_{n-1}\right|\left\|F T_{n} y_{n-1}\right\| \\
& \leq\left(1-\alpha_{n}(v-\rho \gamma)\right)\left\|x_{n}-x_{n-1}\right\| \\
& +\left|\alpha_{n}-\alpha_{n-1}\right|\left(\gamma\left\|V x_{n-1}\right\|+\left\|F T_{n} y_{n-1}\right\|\right) \\
& +\left(1+\mu \alpha_{n-1} L\right) \mathfrak{D}_{B}\left(T_{n}, T_{n-1}\right)+\left|\beta_{n}-\beta_{n-1}\right| M_{1}+a_{n} \\
& \leq\left(1-\alpha_{n}(\nu-\rho \gamma)\right)\left\|x_{n}-x_{n-1}\right\|+\alpha_{n}(\nu-\rho \gamma) \delta_{n},
\end{aligned}
$$

where

$$
\delta_{n}=\frac{1}{(v-\rho \gamma)}\left[\begin{array}{c}
\left(1+\mu \alpha_{n-1} L\right) \frac{\mathfrak{D}_{B}\left(T_{n}, T_{n-1}\right)}{\alpha_{n}} \\
+\left(\left|\frac{\alpha_{n}-\alpha_{n-1}}{\alpha_{n}}\right|+\left|\frac{\beta_{n}-\beta_{n-1}}{\alpha_{n}}\right|\right) M_{2}+\frac{a_{n}}{\alpha_{n}}
\end{array}\right],
$$

and

$$
\sup _{n \geq 1}\left\{\gamma\left\|V x_{n-1}\right\|+\left\|F T_{n} y_{n-1}\right\|, M_{1}\right\} \leq M_{2}
$$

Since $\limsup _{n \rightarrow \infty} \delta_{n} \leq 0$, it follows from Lemma 5, conditions (C2) and (C3) that

$$
\left\|x_{n+1}-x_{n}\right\| \rightarrow 0 \text { as } n \rightarrow \infty
$$


Step 3. Next, we show that $\lim _{n \rightarrow \infty}\left\|x_{n}-T x_{n}\right\|=0$ as $n \rightarrow \infty$. Note that

$$
\begin{aligned}
\left\|x_{n}-T_{n} x_{n}\right\| & \leq\left\|x_{n}-x_{n+1}\right\|+\left\|x_{n+1}-T_{n} x_{n}\right\| \\
& \leq\left\|x_{n}-x_{n+1}\right\|+\left\|P_{C} t_{n}-P_{C} T_{n} x_{n}\right\| \\
& \leq\left\|x_{n}-x_{n+1}\right\|+\left\|\alpha_{n} \rho V x_{n}+\left(I-\alpha_{n} \mu F\right) T_{n} y_{n}-T_{n} x_{n}\right\| \\
& \leq\left\|x_{n}-x_{n+1}\right\|+\left\|\alpha_{n}\left(\rho V x_{n}-\mu F T_{n} y_{n}\right)+T_{n} y_{n}-T_{n} x_{n}\right\| \\
& \leq\left\|x_{n}-x_{n+1}\right\|+\alpha_{n}\left\|\rho V x_{n}-\mu F T_{n} y_{n}\right\|+\left\|y_{n}-x_{n}\right\|+a_{n} \\
& \leq\left\|x_{n}-x_{n+1}\right\|+\alpha_{n}\left\|\rho V x_{n}-\mu F T_{n} y_{n}\right\|+\beta_{n}\left\|S x_{n}-x_{n}\right\|+a_{n} .
\end{aligned}
$$

Since $a_{n} \rightarrow 0$, by using (15) and condition (C1), we obtain

$$
\lim _{n \rightarrow \infty}\left\|x_{n}-T_{n} x_{n}\right\|=0
$$

Hence, we have

$$
\begin{aligned}
\left\|x_{n}-T x_{n}\right\| & \leq\left\|x_{n}-T_{n} x_{n}\right\|+\left\|T_{n} x_{n}-T x_{n}\right\| \\
& \leq\left\|x_{n}-T_{n} x_{n}\right\|+\mathfrak{D}_{B}\left(T_{n}, T\right) \rightarrow 0 \text { as } n \rightarrow \infty
\end{aligned}
$$

Step 4. Next, we show that $\limsup _{n \rightarrow \infty}\left\langle(\rho V-\mu F) x^{*}, x_{n}-x^{*}\right\rangle \leq 0$, where $x^{*}$ is the unique solution of variational inequality (10). Since the sequence $\left\{x_{n}\right\}$ is bounded, it has a weak convergent subsequence $\left\{x_{n_{k}}\right\}$ such that

$$
\limsup _{n \rightarrow \infty}\left\langle(\rho V-\mu F) x^{*}, x_{n}-x^{*}\right\rangle=\limsup _{k \rightarrow \infty}\left\langle(\rho V-\mu F) x^{*}, x_{n_{k}}-x^{*}\right\rangle \text {. }
$$

Let $x_{n_{k}} \rightarrow \widetilde{x}$, as $k \rightarrow \infty$. It follows from Lemma 4 that $\tilde{x} \in$ Fix $(T)=\mathfrak{F}$. Hence

$$
\limsup _{n \rightarrow \infty}\left\langle(\rho V-\mu F) x^{*}, x_{n}-x^{*}\right\rangle=\left\langle(\rho V-\mu F) x^{*}, \tilde{x}-x^{*}\right\rangle \leq 0
$$

Step 5. Now, we show that the sequence $\left\{x_{n}\right\}$ converges strongly to $x^{*}$ as $n \rightarrow \infty$. By using the iteration (9), we have

$$
\begin{aligned}
\left\|x_{n+1}-x^{*}\right\|^{2} & =\left\langle P_{C} t_{n}-x^{*}, x_{n+1}-x^{*}\right\rangle \\
& =\left\langle P_{C} t_{n}-t_{n}, x_{n+1}-x^{*}\right\rangle+\left\langle t_{n}-x^{*}, x_{n+1}-x^{*}\right\rangle .
\end{aligned}
$$

Since the metric projection $P_{C}$ satisfies the inequality

$$
\left\langle x-P_{C} x, y-P_{C} x\right\rangle \leq 0, \forall x \in H, y \in C,
$$

and from (16), we get

$$
\begin{aligned}
\left\|x_{n+1}-x^{*}\right\|^{2} & \leq\left\langle t_{n}-x^{*}, x_{n+1}-x^{*}\right\rangle \\
& =\left\langle\alpha_{n} \rho V x_{n}+\left(I-\alpha_{n} \mu F\right) T_{n} y_{n}-x^{*}, x_{n+1}-x^{*}\right\rangle \\
& =\left\langle\alpha_{n}\left(\rho V x_{n}-\mu F x^{*}\right)+\left(I-\alpha_{n} \mu F\right) T_{n} y_{n}\right. \\
& \left.-\left(I-\alpha_{n} \mu F\right) T_{n} x^{*}, x_{n+1}-x^{*}\right\rangle \\
& =\alpha_{n} \rho\left\langle V x_{n}-V x^{*}, x_{n+1}-x^{*}\right\rangle+\alpha_{n}\left\langle\rho V x^{*}-\mu F x^{*}, x_{n+1}-x^{*}\right\rangle \\
& +\left\langle\left(I-\alpha_{n} \mu F\right) T_{n} y_{n}-\left(I-\alpha_{n} \mu F\right) T_{n} x^{*}, x_{n+1}-x^{*}\right\rangle .
\end{aligned}
$$


Hence, from (11) and Lemma 3, we obtain

$$
\begin{aligned}
\left\|x_{n+1}-x^{*}\right\|^{2} & \leq \alpha_{n} \rho \gamma\left\|x_{n}-x^{*}\right\|\left\|x_{n+1}-x^{*}\right\|+\alpha_{n}\left\langle\rho V x^{*}-\mu F x^{*}, x_{n+1}-x^{*}\right\rangle \\
& +\left(1-\alpha_{n} v\right)\left(\left\|y_{n}-x^{*}\right\|+a_{n}\right)\left\|x_{n+1}-x^{*}\right\| \\
& \leq \alpha_{n} \rho \gamma\left\|x_{n}-x^{*}\right\|\left\|x_{n+1}-x^{*}\right\|+\alpha_{n}\left\langle\rho V x^{*}-\mu F x^{*}, x_{n+1}-x^{*}\right\rangle \\
& +\left(1-\alpha_{n} v\right)\left(\left\|x_{n}-x^{*}\right\|+\beta_{n}\left\|S x^{*}-x^{*}\right\|+a_{n}\right)\left\|x_{n+1}-x^{*}\right\| \\
& =\left(1-\alpha_{n}(v-\rho \gamma)\right)\left\|x_{n}-x^{*}\right\|\left\|x_{n+1}-x^{*}\right\| \\
& +\alpha_{n}\left\langle\rho V x^{*}-\mu F x^{*}, x_{n+1}-x^{*}\right\rangle \\
& +\left(1-\alpha_{n} v\right) \beta_{n}\left\|S x^{*}-x^{*}\right\|\left\|x_{n+1}-x^{*}\right\| \\
& +\left(1-\alpha_{n} v\right) a_{n}\left\|x_{n+1}-x^{*}\right\| \\
& \leq \frac{\left(1-\alpha_{n}(v-\rho \gamma)\right)}{2}\left(\left\|x_{n}-x^{*}\right\|^{2}+\left\|x_{n+1}-x^{*}\right\|^{2}\right) \\
& +\alpha_{n}\left\langle\rho V x^{*}-\mu F x^{*}, x_{n+1}-x^{*}\right\rangle+\beta_{n}\left\|S x^{*}-x^{*}\right\|\left\|x_{n+1}-x^{*}\right\| \\
& +a_{n}\left\|x_{n+1}-x^{*}\right\|,
\end{aligned}
$$

which implies that

$$
\begin{aligned}
\left\|x_{n+1}-x^{*}\right\|^{2} & \leq \frac{\left(1-\alpha_{n}(v-\rho \gamma)\right)}{\left(1+\alpha_{n}(v-\rho \gamma)\right)}\left\|x_{n}-x^{*}\right\|^{2} \\
& +\frac{2 \alpha_{n}}{\left(1+\alpha_{n}(v-\rho \gamma)\right)}\left\langle\rho V x^{*}-\mu F x^{*}, x_{n+1}-x^{*}\right\rangle \\
& +\frac{2 \beta_{n}}{\left(1+\alpha_{n}(v-\rho \gamma)\right)}\left\|S x^{*}-x^{*}\right\|\left\|x_{n+1}-x^{*}\right\| \\
& +\frac{2 a_{n}}{\left(1+\alpha_{n}(v-\rho \gamma)\right)}\left\|x_{n+1}-x^{*}\right\| \\
& \leq\left(1-\alpha_{n}(v-\rho \gamma)\right)\left\|x_{n}-x^{*}\right\|^{2}+\alpha_{n}(v-\rho \gamma) \theta_{n}
\end{aligned}
$$

where

$$
\theta_{n}=\frac{2 \alpha_{n}}{\left(1+\alpha_{n}(v-\rho \gamma)\right)(v-\rho \gamma)}\left[\begin{array}{c}
\left\langle\rho V x^{*}-\mu F x^{*}, x_{n+1}-x^{*}\right\rangle+\frac{\beta_{n}}{\alpha_{n}} M_{3} \\
+\frac{a_{n}}{\alpha_{n}}\left\|x_{n+1}-x^{*}\right\|
\end{array}\right]
$$

and

$$
\sup _{n \geq 1}\left\{\left\|S x^{*}-x^{*}\right\|\left\|x_{n+1}-x^{*}\right\|\right\} \leq M_{3}
$$

Since $\frac{\beta_{n}}{\alpha_{n}} \rightarrow 0$ and $\frac{a_{n}}{\alpha_{n}} \rightarrow 0$, we get

$$
\limsup _{n \rightarrow \infty} \theta_{n} \leq 0
$$

So, it follows from Lemma 5 that the sequence $\left\{x_{n}\right\}$ generated by (9) converges strongly to $x^{*} \in \mathfrak{F}$ which is the unique solution of variational inequality (10).

Step 6. Finally, since the point $x^{*}$ is the unique solution of variational inequality (10), in particular if we take $V=0$ and $F=I$ in the variational inequality (10), then we get

$$
\left\langle-\mu x^{*}, x-x^{*}\right\rangle \leq 0, \forall x \in \mathfrak{F} .
$$

So we have

$$
\left\langle x^{*}, x^{*}-x\right\rangle=\left\langle x^{*}, x^{*}\right\rangle-\left\langle x^{*}, x\right\rangle \leq 0 \Longrightarrow\left\|x^{*}\right\|^{2} \leq\left\|x^{*}\right\|\|x\| .
$$


Hence, $x^{*}$ is the unique solution to the quadratic minimization problem $x^{*}=\arg _{\min } n_{x \in \mathfrak{F}}\|x\|^{2}$. This completes the proof.

From Theorem 1, we can deduce the following interesting corollaries.

Corollary 1. Let $C$ be a nonempty closed convex subset of a real Hilbert space $H$. Let $S: C \rightarrow H$ be a nonexpansive mapping and $\left\{T_{n}\right\}$ be a sequence of nonexpansive mappings such that $\mathfrak{F} \neq \varnothing$. Suppose that $T x=\lim _{n \rightarrow \infty} T_{n} x$ for all $x \in C$. Let $V: C \rightarrow H$ be a $\gamma$-Lipschitzian mapping, $F: C \rightarrow H$ be a L-Lipschitzian and $\eta$-strongly monotone operator such that these coefficients satisfy $0<\mu<\frac{2 \eta}{L^{2}}, 0 \leq \rho \gamma<v$, where $v=1-\sqrt{1-\mu\left(2 \eta-\mu L^{2}\right)}$. For an arbitrarily initial value $x_{1} \in C$, consider the sequence $\left\{x_{n}\right\}$ in $C$ generated by (9) where $\left\{\alpha_{n}\right\}$ and $\left\{\beta_{n}\right\}$ are sequences in $[0,1]$ satisfying the conditions (C1)-(C3) of Theorem 1 except the condition $\lim _{n \rightarrow \infty} \frac{a_{n}}{\alpha_{n}}=0$. Then, the sequence $\left\{x_{n}\right\}$ converges strongly to $x^{*} \in \mathfrak{F}$, where $x^{*}$ is the unique solution of variational inequality (10).

Let $\lambda_{i}>0(i=1,2,3, \ldots N)$ such that $\sum_{i=1}^{N} \lambda_{i}=1$ and $T_{1}, T_{2}, \ldots T_{N}$ be nonexpansive self mappings on $C$ such that $\bigcap_{i=1}^{N} \operatorname{Fix}\left(T_{i}\right) \neq \varnothing$. Then, $\sum_{i=1}^{N} \lambda_{i} T_{i}$ is nonexpansive self mapping on $C$ (see [23, Proposition 6.1]).

Corollary 2. Let $C$ be a nonempty closed convex subset of a real Hilbert space $H$. Let $\lambda_{i}>0(i=1,2,3, \ldots N)$ such that $\sum_{i=1}^{N} \lambda_{i}=1$ and $S, T_{1}, T_{2}, \ldots T_{N}$ be nonexpansive self mappings on $C$ such that $\bigcap_{i=1}^{N} F i x\left(T_{i}\right) \neq \varnothing$. Let $V: C \rightarrow H$ be a $\gamma$-Lipschitzian mapping, $F: C \rightarrow H$ be a L-Lipschitzian and $\eta$-strongly monotone operator such that these coefficients satisfy $0<\mu<\frac{2 \eta}{L^{2}}, 0 \leq \rho \gamma<v$, where $v=1-\sqrt{1-\mu\left(2 \eta-\mu L^{2}\right)}$. For an arbitrarily initial value $x_{1} \in C$, consider the sequence $\left\{x_{n}\right\}$ in $C$ generated by

$$
\left\{\begin{array}{l}
y_{n}=\beta_{n} S x_{n}+\left(1-\beta_{n}\right) x_{n} \\
x_{n+1}=P_{C}\left[\alpha_{n} \rho V x_{n}+\left(I-\alpha_{n} \mu F\right) \sum_{i=1}^{N} \lambda_{i} T_{i} y_{n}\right], \forall n \geq 1
\end{array}\right.
$$

where $\left\{\alpha_{n}\right\}$ and $\left\{\beta_{n}\right\}$ are sequences in $[0,1]$ satisfying the conditions (C1) and (C2) of Theorem 1 except the condition $\lim _{n \rightarrow \infty} \frac{a_{n}}{\alpha_{n}}=0$. Then, the sequence $\left\{x_{n}\right\}$ in $C$ generated by (17) converges strongly to $x^{*} \in \bigcap_{i=1}^{N}$ Fix $\left(T_{i}\right)$, where $x^{*}$ is the unique solution of variational inequality

$$
\left\langle(\rho V-\mu F) x^{*}, x-x^{*}\right\rangle \leq 0, \forall x \in \bigcap_{i=1}^{N} \operatorname{Fix}\left(T_{i}\right) .
$$

Remark. Our results can be reduced to some corresponding results in the following ways:

(1) In our iterative process (9), if we take $S=I$ ( $I$ is the identity operator of $C$ ), then we derive the iterative process (7) which is studied by Sahu et. al. [19]. Therefore, Theorem 1 generalizes the main result of Sahu et. al. [19, Theorem 3.1]. Also, Corollary 1 and Corollary 2 extends the Corollary 3.4 and Theorem 4.1 of Sahu et. al. [19], respectively. So, our results extends the corresponding results of Ceng et. al. [17] and of many other authors.

(2) If we take $S$ as a nonexpansive self mapping on $C$ and $T_{n}=T$ for all $n \geq 1$ such that $T$ is a nonexpansive mapping in (9), then we get the iterative process (8) of Wang and Xu. [20]. Hence, Theorem 1 generalizes the main result of Wang and $\mathrm{Xu}$ [20, Theorem 3.1]. So, our results extend and improve the corresponding results of [4, ?].

(3) The problem of finding the solution of variational inequality (10), is equivalent to finding the solutions of hierarchical fixed point problem

$$
\left\langle(I-S) x^{*}, x^{*}-x\right\rangle \leq 0, \forall x \in \mathfrak{F}
$$

where $S=I-(\rho V-\mu F)$.

\section{References}

[1] R. P. Agarwal, D. O’Regan, and D. R. Sahu, Iteratıve Constructıon of Fixed Points of Nearly Asymptotıcally Nonexpansıve Mappıngs, Journal of Nonlinear and Convex Analysis, Vol.8, No.1, 61-79, 2007. 
[2] R. P. Agarwal, D. O’Regan, and D. R. Sahu, Fixed Point Theory for Lipschitzian-Type Mappings with Applications, Topological Fixed Point Theory and Its Applications, Springer, New York, NY, USA, 2009.

[3] F. Cianciaruso, G. Marino, L. Muglia, Y. Yao, On a two-steps algorithm for hierarchical fixed point problems and variational inequalities. J. Inequal. Appl., 1-13, 2009.

[4] M. Tian, A general iterative algorithm for nonexpansive mappings in Hilbert spaces, Nonlinear Analysis, Theory, Methods and Applications, vol. 73, no. 3, 689-694, 2010.

[5] Y. Yao, Y.J. Cho, Y.C. Liou, Iterative algorithms for hierarchical fixed points problems and variational inequalities. Math. Comput. Model. 52 (9-10), 1697-1705, 2010.

[6] G. Gu, S. Wang, Y.J. Cho, Strong convergence algorithms for hierarchical fixed points problems and variational inequalities. J. Appl. Math. 2011, 1-17, 2011

[7] Y. Yao, R. Chen, Regularized algorithms for hierarchical fixed-point problems, Nonlinear Analysis, 74, 6826-6834, 2011.

[8] M, Tian and L.H. Huang, Iterative methods for constrained convex minimization problem in Hilbert spaces, Fixed Point Theory and Applications, 2013:105, 2013.

[9] I. Yamada, The hybrid steepest-descent method for variational inequality problems over the intersection of the fixed point sets of nonexpansive mappings. In: Butnariu, D, Censor, Y, Reich, S (eds.) Inherently Parallel Algorithms and Optimization and Their Applications, pp. 473-504. North-Holland, Amsterdam, 2001.

[10] A. Moudafi, Krasnoselski-Mann iteration for hierarchical fixed-point problems. Inverse Probl. 23 (4), 1635-1640, 2007.

[11] P.E. Mainge, A, Moudafi, Strong convergence of an iterative method for hierarchical fixed-point problems. Pac. J. Optim. 3 (3), 529-538, 2007.

[12] Y. Yao and Y. C. Liou, Weak and strong convergence of Krasnoselski-Mann iteration for hierarchical fixed point problems, Inverse Problems 24, 015015, 8pp, 2008,

[13] H.K. Xu, Viscosity method for hierarchical fixed point approach to varıatıonal inequalitıes, Taiwanese Journal of Mathematıcs, Vol. 14, No. 2, 463-478, 2010.

[14] G. Marino and H.K. Xu, Explicit hierarchical fixed point approach to variational inequalities. J. Optim. Theory Appl. 149 (1), $61-78,2011$.

[15] A. Bnouhachem and M.A. Noor, An iterative method for approximating the common solutions of a variational inequality, a mixed equilibrium problem and a hierarchical fixed point problem, Journal of Inequalities and Applications, 2013:490, 2013.

[16] G. Marino and H.K. Xu, A general iterative method for nonexpansive mappings in Hilbert spaces, J. Math. Anal. Appl., 318, 43-52, 2006.

[17] L.C. Ceng, Q.H. Ansari and J.C. Yao, Some iterative methods for finding fixed points and for solving constrained convex minimization problems. Nonlinear Analysis, 74, 5286-5302, 2011.

[18] N.C. Wong, D.R. Sahu and J.C. Yao, A generalized hybrid steepest-descent method for variational inequalities in Banach spaces, Fixed Point Theory and Applications, vol. 2011, Article ID 754702, 28 pages, 2011.

[19] D.R. Sahu, S.M. Kang and V. Sagar, Approximation of Common Fixed Points of a Sequence of Nearly Nonexpansive Mappings and Solutions of Variational Inequality Problems, Journal of Applied Mathematics, Article ID 902437, 12 pages, 2012.

[20] Y. Wang and W. Xu, Strong convergence of a modified iterative algorithm for hierarchical fixed point problems and variational inequalities, Fixed Point Theory and Applications, 2013:121, 2013.

[21] K. Goebel, W. A. Kirk, Topics on Metric Fixed-Point Theory, Cambridge University Press, Cambridge, England, 1990.

[22] H.K. Xu and T.H.Kim, Convergence of hybrid steepest-descent methods for variational inequalities," Journal of Optimization Theory and Applications, vol. 119, no. 1, 185-201, 2003.

[23] N.C. Wong, D.R. Sahu and J.C. Yao, Solving variational inequalities involving nonexpansive type mappings, Nonlinear Analysis, Theory, Methods and Applications, vol. 69, no. 12, 4732-4753, 2008.

[24] Hu, H. Y., \& Ceng, L. C. (2015). A hybrid iterative method for a common solution of variational inequalities, generalized mixed equilibrium problems, and hierarchical fixed point problems. Journal of Inequalities and Applications, 2015(1), 1-29.

[25] Bnouhachem, A. (2014). A modified projection method for a common solution of a system of variational inequalities, a split equilibrium problem and a hierarchical fixed-point problem. Fixed Point Theory and Applications, 2014(1), 1-25.

[26] Karahan, I., \& Ozdemir, M. (2014). Convergence Theorems for Hierarchical Fixed Point Problems and Variational Inequalities. Journal of Applied Mathematics, 2014.

[27] Karahan, I., Secer, A., Ozdemir, M., \& Bayram, M. (2015). The common solution for a generalized equilibrium problem, a variational inequality problem and a hierarchical fixed point problem. Journal of Inequalities and Applications, 2015(1), 1-25.

[28] Bnouhachem, A., Al-Homidan, S., \& Ansari, Q. H. (2014). An iterative method for common solutions of equilibrium problems and hierarchical fixed point problems. Fixed Point Theory and Applications, 2014(1), 194. 